\title{
Airway stenting in excessive central airway collapse
}

\author{
Mihir Parikh, Jennifer Wilson, Adnan Majid, Sidhu Gangadharan \\ Division of Thoracic Surgery and Interventional Pulmonology, Beth Israel Deaconess Medical Center, Boston, MA, USA \\ Contributions: (I) Conception and design: All authors; (II) Administrative support: All authors; (III) Provision of study materials or patients: All \\ authors; (IV) Collection and assembly of data: All authors; (V) Data analysis and interpretation: All authors; (VI) Manuscript writing: All authors; (VII) \\ Final approval of manuscript: All authors. \\ Correspondence to: Mihir Parikh. Division of Thoracic Surgery and Interventional Pulmonology, Beth Israel Deaconess Medical Center, 185 Pilgrim \\ Road, Deac 201, Boston, MA 02215, USA. Email: msparikh@bidmc.harvard.edu.
}

\begin{abstract}
In tracheobronchomalacia (TBM) and other disorders, weakened airway walls lead to expiratory central airway collapse (ECAC) and can cause symptoms of cough, dyspnea, retained secretions, and recurrent pulmonary infections. Diagnosis of severe ECAC is based on the presence of $>90 \%$ expiratory airway collapse on dynamic computed tomography (CT) and/or bronchoscopy. We offer patients with severe ECAC a trial of airway stenting to determine whether splinting of the central airways leads to improvements in symptoms, quality of life, exercise capacity, or respiratory function. Patients who respond positively to airway stenting are considered for tracheobronchoplasty.
\end{abstract}

Keywords: Tracheobronchomalacia (TBM); excessive dynamic airway collapse (EDAC); excessive central airway collapse (ECAC); tracheobronchoplasty; airway stenting

Received: 11 February 2017; Accepted: 18 October 2017; Published: 22 November 2017. doi: 10.21037 /jovs.2017.10.12

View this article at: http://dx.doi.org/10.21037/jovs.2017.10.12

\section{Introduction}

Excessive central airway collapse (ECAC) is an underrecognized syndrome characterized by excessive collapse and obstruction of the trachea and bronchi with expiration. ECAC includes tracheobronchomalacia (TBM) and excessive dynamic airway collapse (EDAC). TBM is defined by weakening of airway walls leading to collapse of lateral and anterior cartilaginous structures in the airways. In contrast, EDAC occurs when airway lumen is reduced due to excessive bowing of the posterior membrane on expiration. While the pathophysiology and morphology of TBM and EDAC are distinct, their clinical symptoms and management are similar. Patients with ECAC often present with symptoms of dyspnea, cough, wheezing, and frequent respiratory infections. Therapeutic options for ECAC are limited and include non-invasive therapies, such as continuous positive airway pressure (CPAP) or bi-level positive airway pressure (BiPAP), and more invasive options, including stent placement and tracheobronchoplasty (1). Here, we discuss the role of endobronchial stents in the management of ECAC.

\section{Patient selection and workup}

The exact prevalence of ECAC in adults remains unknown. This is partly due to persistent debate over diagnostic criteria for ECAC. The standard definition requires a greater than $50 \%$ reduction in airway cross-sectional area (CSA) with expiration for diagnosis of ECAC. This definition, however, is overly broad with as many as $78 \%$ of healthy, asymptomatic volunteers exceeding the threshold of $50 \%$ collapse of the airways (2). At our institution, we have increased the threshold to $70 \%$ reduction in CSA for diagnosis of ECAC based on evidence indicating that expiratory central airway collapse (ECAC) in normal individuals is $54 \%$ to $56 \%$. A $70 \%$ reduction threshold is approximately one standard deviation higher than these normal controls (2). We limit treatment to those patients having severe ECAC, defined as a reduction of $>90 \%$ in the CSA of the affected airways. ECAC can either be a congenital or an acquired condition. The acquired form can 
be associated with traumatic injuries, prior tracheostomy or endotracheal intubation, or inflammatory conditions, such as relapsing polychondritis. In many patients, no clear cause is identified and their ECAC is classified as idiopathic. ECAC can also be classified based on the shape of the airways with dynamic respiratory maneuvers and based on whether airway collapse is due to weakness of the anterior cartilaginous rings or the posterior membrane (3). Patients with frown-shaped airways on expiration and lunate airways on inspiration are most amenable to surgical intervention with tracheobronchoplasty (4).

Diagnosis of ECAC is based on identification of dynamic airway collapse with computed tomography (CT) scanning or direct visualization of airway collapse on bronchoscopy with forced expiratory maneuvers (5). Pulmonary function tests (PFTs) can show a range of patterns in patients with ECAC, including restrictive and obstructive ventilatory defects. Moreover, obstructive changes on PFTs can be due to concomitant obstructive lung disease, such as chronic obstructive pulmonary disease (COPD) or asthma, and not due to the ECAC itself (6). In many patients, the diagnosis and subsequent management of ECAC can be confounded by the presence of other pulmonary comorbidities, including COPD, asthma, laryngopharyngeal reflux (LPR), vocal cord dysfunction, and obstructive sleep apnea $(7,8)$. Management options are limited. Medical treatments, including bronchodilators or inhaled corticosteroids, can provide some relief, particularly in patients with comorbid COPD or asthma. Mucociliary clearance techniques, including mucolytics and flutter valves, can assist patients with significant retained secretions. Pursed lip breathing and non-invasive positive pressure ventilation, including CPAP and BiPAP, can temporarily improve expiratory airflow to some degree. Invasive options to maintain or restore airway patency include tracheostomy or T-tube placement, stent placement and surgical intervention with tracheobronchoplasty (9). Airway stents predominantly have a role as trial therapy to determine the likelihood of successful symptom improvement with tracheobronchoplasty $(10,11)$. Once a patient is diagnosed with severe ECAC and is being considered for definitive surgical intervention at our institution, we initiate a stent trial. We place tracheal and bronchial stents at sites of severe malacia (greater than $90 \%$ collapse of the airway with expiration). After a 2-week trial, patients are assessed for improvement in quality of life or in symptoms thought to be related to their ECAC. PFTs and 6-minute walk test (6MWT) are performed before and after stent placement to assess for objective improvements in respiratory function and exercise capacity, as measured by changes in forced vital capacity (FVC), expiratory volume during the first second of forced expiration (FEV1), and distance walked during 6MWT. The stents are removed within 2 weeks, and patients with subjective or objective improvement during the stent trial proceed to evaluation for tracheobronchoplasty (Figure 1).

Based on our experience, approximately $60 \%$ to $75 \%$ of patients with ECAC will experience symptomatic improvement during the stent trial. Of those patients who responded positively to the stent trial, $80 \%$ will have improvements in quality of life after tracheobronchoplasty $(12,13)$. Stent placement leads to improvements in respiratory symptoms, quality of life, and functional status in patients with ECAC (10). Despite limited data on longterm outcomes and complications after stent placement, our preference is to avoid definitive treatment of ECAC by stenting in patients who are able to undergo definitive surgical treatment because many patients will develop a stent-related complication after approximately 2 weeks. Patients treated with airway stents for long term airway stabilization can develop significant granulation tissue at the proximal and distal ends of the stent leading to worsening airway stenosis and symptoms $(10,13)$.

Some patients will experience significant improvements during a stent trial but will be deemed poor surgical candidates for tracheobronchoplasty or will choose to defer surgical intervention. Surgical candidacy is based on a global assessment based on a patient's perceived fitness for surgery, individualized risk of surgery, and potential benefit gained from surgery. Patients may be unfit for surgery due to medical comorbidities or severely diminished FEV1/DLCO. Patients who are not surgical candidates must have optimal management of their medical comorbidities with maximal medical therapy for their ECAC. This can include noninvasive ventilation. If symptoms persist, then airway stents can be placed and maintained in these patients to restore airway patency for long-term definitive management but this can be a very arduous and often unsuccessful course.

\section{Equipment preferences}

We utilize both silicone and uncovered self-expanding metallic stents (SEMS) in our patients with ECAC. A Y-shaped silicone stent can be placed in patients with concomitant severe malacia in the distal trachea and bilateral main stem bronchi. These require rigid bronchoscopy for 


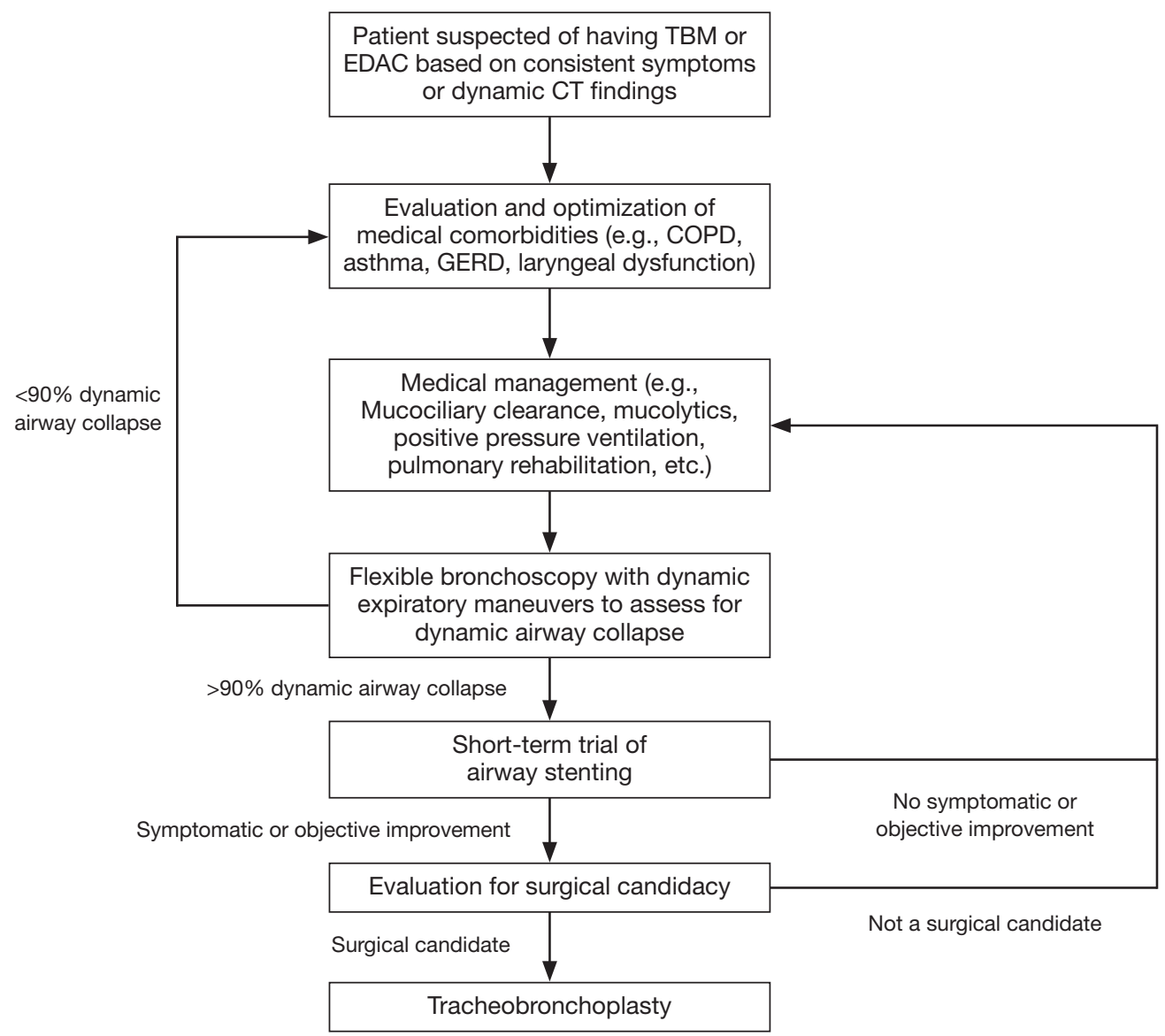

Figure 1 Management pathway for patients with ECAC. ECAC, excessive central airway collapse.

placement and can be prone to mucous plugging. In addition, a subset of patients will develop intractable coughing with these Y stents. However, they are easily removed and can be custom tailored to fit individual patient specifications.

Utilization of SEMS for benign disease generally should be avoided despite the ease of placement. While the elastic properties of Nitinol-based SEMS allow them to conform to the shape of complex airways and to maintain their shape with cough and uncovered SEMS may preserve mucociliary clearance, there can be significant disadvantages to SEMS. These have been the subject of an FDA notification which warns about complications such as fracture, migration, granulation tissue, stenosis, and infection when SEMS are used to treat benign disease $(14,15)$. With this caveat in mind, we have found that for short-term trial usage the complications rates for SEMS in ECAC are acceptably low. Therefore, as an initial intervention to assess a patient's candidacy for surgical tracheobronchoplasty we occasionally have utilized SEMS, especially in patients for whom cough may be their predominant complaint and may have the assessment of the benefit of airway stabilization confounded by a silicone $Y$ stent-related cough $(16,17)$.

\section{Procedure}

Prior to stent placement, patients with suspected ECAC are evaluated with dynamic flexible bronchoscopy under moderate sedation and sites of severe malacia (greater than $90 \%$ dynamic collapse) are identified with forced expiratory maneuvers (Figure 2). Stents are placed in the trachea, both main stem bronchi, and right bronchus intermedius at these sites of severe malacia. Stent placement in more distal segments increases the risk of mucous plugging and stent obstruction. The diameter of the stent is determined by measurements of airway diameter on CT scan and by direct measurement using an Aero Sizer tracheobronchial stent sizing device (Merit Medical) during flexible bronchoscopy. Stent length is based on the length of the malacic airway 


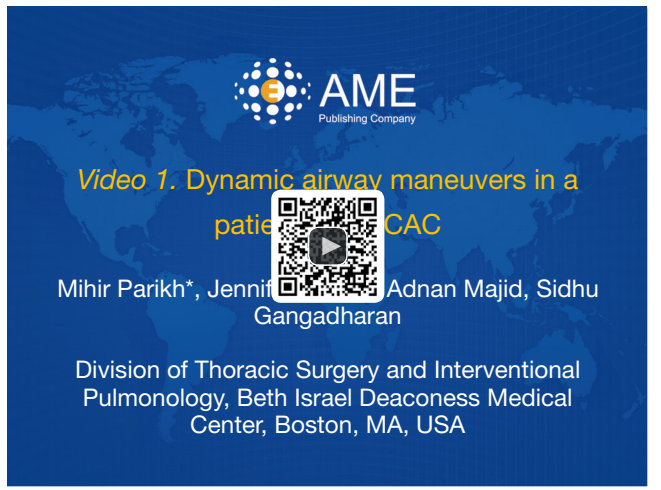

Figure 2 Dynamic airway maneuvers in a patient with ECAC (18). ECAC, excessive central airway collapse. Available online: http://www.asvide.com/articles/1828

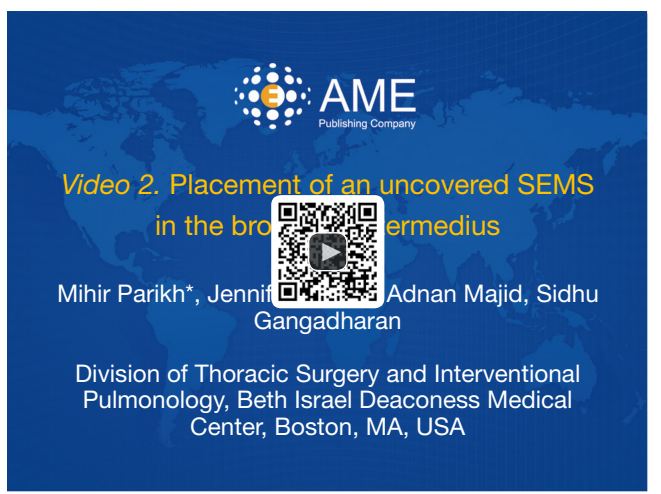

Figure 3 Placement of an uncovered SEMS in the bronchus intermedius (19). SEMS, self-expanding metallic stent. Available online: http://www.asvide.com/articles/1829

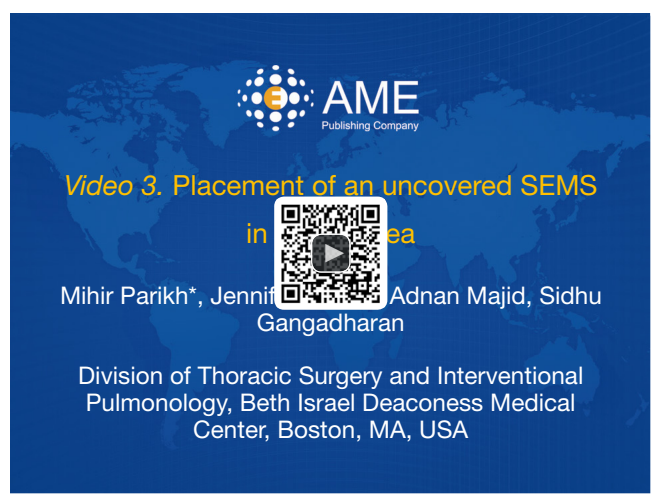

Figure 4 Placement of an uncovered SEMS in the trachea (20). SEMS, self-expanding metallic stent.

Available online: http://www.asvide.com/articles/1830

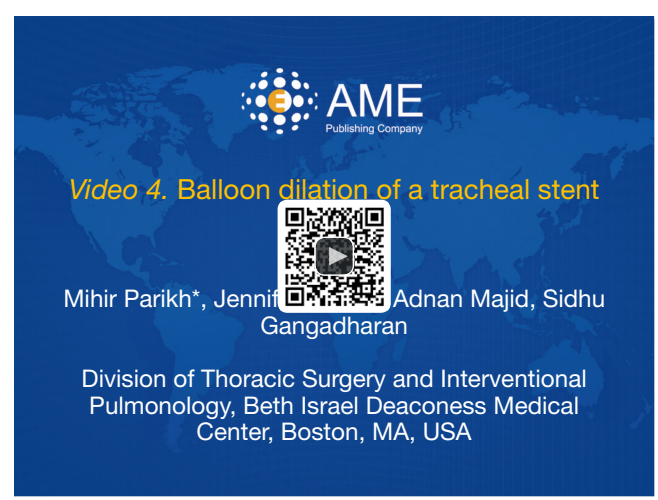

Figure 5 Balloon dilation of a tracheal stent (21). Available online: http://www.asvide.com/articles/1831

segment. We use the shortest stent length possible to minimize the impact on mucociliary clearance. We prefer to use uncovered SEMS as these tend to minimize the risk of mucous plugging, a common stent-related complication that can confound interpretation of the stent trial. We use silicone Y-stents if airway collapse is focused at the main carina or if the airway diameter is greater than $20 \mathrm{~mm}$, which is the diameter of the largest uncovered selfexpanding airway metallic stent we carry.

In most patients our practice is to place stents under rigid bronchoscopy. Y-silicone stents are placed into the malacic segments using the dedicated stent deployment device. Their position can then be adjusted using the rigid forceps. For SEMS, the rigid bronchoscope is positioned just proximal to the target airway segment and the stent is introduced through the rigid bronchoscope. The flexible bronchoscope or rigid camera is then introduced into the rigid bronchoscope and the SEMS is positioned and subsequently deployed into the target airway under direct visualization (Figures 3,4). Finally, we inflate a CRE Endovention Balloon (Boston Scientific) within the stent to achieve maximal deployment in the airway (Figure 5).

\section{Role of team members}

At our institution, the care of patients with ECAC is shared amongst a multi-disciplinary team of thoracic surgeons, interventional pulmonologists, laryngologists, gastroenterologists, chest radiologists, anesthesiologists, nurses, and physical therapists. Patients are initially evaluated by our thoracic surgeons and interventional pulmonologists to assess the presence and severity of symptoms attributable to ECAC as well as to optimize management of comorbid 
conditions, such as COPD, asthma, and obstructive sleep apnea. We also routinely refer patients to our colleagues in laryngology and gastroenterology to screen for gastroesophageal reflux, LPR and vocal cord dysfunction. Stenting and tracheobronchoplasty procedures are performed with the assistance of anesthesiologists and nurses with particular training in thoracic procedures, including the use of rigid bronchoscopy and jet ventilation.

\section{Post-operative management}

Complications of stent placement include those attendant to bronchoscopy and general anesthesia. These include airway obstruction, bleeding, pneumothorax, pneumomediastinum, and airway perforation (15). Post-procedure respiratory distress is of particular concern in patients with ECAC given their pre-existing central airway compromise and their tendency to develop intractable cough and bronchospasm. Stents, particularly non Y silicone stents, can also migrate in the airways. After stent placement, patients can also experience worsening cough, increased sputum production, mucous impaction, and ingrowth of granulation tissue with subsequent airway narrowing. These adverse sequelae can confound a stent trial and limit the ability to predict successful outcome after tracheobronchoplasty (4).

Post-stent care is designed to minimize these adverse sequelae. Mucociliary clearance techniques are of vital importance to minimize the risk of mucous plugging and infection. Patients should be treated with daily mucolytics and are instructed to utilize a flutter valve or other oral oscillatory device. Airway humidification is achieved with daily treatment with nebulized saline. Nebulized acetylcysteine can be used in patients suffering from excessively thick or copious respiratory secretions. We remove silicone and SEMS under rigid bronchoscopy using rigid forceps. The most common complications after stent removal are respiratory distress secondary to laryngospasm, severe cough, or transient worsening of other ECAC-related symptoms. To minimize the risk of laryngospasm and cough we administer $15 \mathrm{~mL}$ of $1 \%$ lidocaine upon removal of the rigid bronchoscope at the vocal cords $(5 \mathrm{~mL})$ and the tracheobronchial tree $(10 \mathrm{~mL})$. In addition, we use NIV in the immediate post-operative period to mitigate the degree of central airway collapse.

\section{Conclusions}

ECAC, including the syndrome of TBM, is an under- recognized condition characterized by abnormal dynamic collapse of the central airways leading to cough, dyspnea, retained secretions, and recurrent infections. In patients with ECAC, restoration of airway patency with airway stenting can serve as a therapeutic trial to assess candidacy for tracheobronchoplasty or, in selected high-risk patients, as a long-term definitive management strategy. Airway stenting is a relatively safe procedure when performed by an experienced bronchoscopist, but appropriate patient selection and complex post-operative management require coordinated care by a dedicated team of thoracic surgeons, interventional pulmonologists, and others.

\section{Acknowledgements}

None.

\section{Footnote}

Conflicts of Interest: The authors have no conflicts of interest to declare.

Informed Consent: Written informed consent was obtained for the recording and publication of patient videos in this manuscript. Identifying information, including names, initials, and hospital numbers, have been removed from all associated materials.

\section{References}

1. Murgu S, Colt H. Tracheobronchomalacia and excessive dynamic airway collapse. Clin Chest Med 2013;34:527-55.

2. Boiselle PM, O'Donnell CR, Bankier AA, et al. Tracheal collapsibility in healthy volunteers during forced expiration: assessment with multidetector CT. Radiology 2009;252:255-62.

3. Ridge CA, O'donnell CR, Lee EY, et al. Tracheobronchomalacia: current concepts and controversies. J Thorac Imaging 2011;26:278-89.

4. Gangadharan SP. Tracheobronchomalacia in adults. Semin Thorac Cardiovasc Surg 2010;22:165-73.

5. Majid A, Gaurav K, Sanchez JM, et al. Evaluation of tracheobronchomalacia by dynamic flexible bronchoscopy. A pilot study. Ann Am Thorac Soc 2014;11:951-5.

6. Majid A, Sosa AF, Ernst A, et al. Pulmonary function and flow-volume loop patterns in patients with tracheobronchomalacia. Respir Care 2013;58:1521-6.

7. Nataraj D, Majid A, Chuttani R, et al. Prevalence 
of gastroesophageal rflux in patients with tracheobronchomalacia. Chest 2009;136:80S.

8. Ochs RA, Petkovska I, Kim HJ, et al. Prevalence of tracheal collapse in an emphysema cohort as measured with end-expiration CT. Acad Radiol 2009;16:46-53.

9. Murgu SD, Colt HG. Treatment of adult tracheobronchomalacia and excessive dynamic airway collapse: an update. Treat Respir Med 2006;5:103-15.

10. Ernst A, Majid A, Feller-Kopman D, et al. Airway stabilization with silicone stents for treating adult tracheobronchomalacia: a prospective observational study. Chest 2007;132:609-16.

11. Ochoa S, Cheng GZ, Folch E, et al. Use of Self-expanding Metallic Airway Stents in Tracheobronchomalacia. J Bronchology Interv Pulmonol 2015;22:e9-e11.

12. Majid A, Guerrero J, Gangadharan S, et al. Tracheobronchoplasty for severe tracheobronchomalacia: a prospective outcome analysis. Chest 2008;134:801-7.

13. Ernst A, Odell DD, Michaud G, et al. Central airway stabilization for tracheobronchomalacia improves quality of life in patients with COPD. Chest 2011;140:1162-8.

14. Lund ME, Force S. Airway stenting for patients with benign airway disease and the Food and Drug Administration Advisory: A Call for Restraint. Chest 2007;

doi: 10.21037/jovs.2017.10.12

Cite this article as: Parikh M, Wilson J, Majid A, Gangadharan S. Airway stenting in excessive central airway collapse. J Vis Surg 2017;3:172.
132:1107-8.

15. Casal RF. Update in airway stents. Curr Opin Pulm Med 2010;16:321-8.

16. Majid A, Alape D, Kheir F, et al. Short-Term Use of Uncovered Self-Expanding Metallic Airway Stents for Severe Expiratory Central Airway Collapse. Respiration 2016;92:389-96.

17. Murgu SD, Egressy K, Laxmanan B, et al. Central Airway Obstruction: Benign Strictures, Tracheobronchomalacia, and Malignancy-related Obstruction. Chest 2016;150:426-41.

18. Parikh M, Wilson J, Majid A, Gangadharan S. Dynamic airway maneuvers in a patient with ECAC. Asvide 2017;4:509. Available online: http://www.asvide.com/ articles/1828

19. Parikh M, Wilson J, Majid A, Gangadharan S. Placement of an uncovered SEMS in the bronchus intermedius. Asvide 2017;4:510. Available online: http://www.asvide. com/articles/1829

20. Parikh M, Wilson J, Majid A, Gangadharan S. Placement of an uncovered SEMS in the trachea. Asvide 2017;4:511. Available online: http://www.asvide.com/articles/1830

21. Parikh M, Wilson J, Majid A, Gangadharan S. Balloon dilation of a tracheal stent. Asvide 2017;4:512. Available online: http://www.asvide.com/articles/1831 